\title{
LIVER
}

\section{Genotype C hepatitis B virus infection is associated with an increased risk of hepatocellular carcinoma}

\author{
H L-Y Chan, A Y Hui, M L Wong, A M-L Tse, L C-T Hung, V W-S Wong, J J-Y Sung
}

Gut 2004;53:1494-1498. doi: 10.1136/gut.2003.033324

See end of article for authors' affiliations

.....................

Correspondence to: Dr H L-Y Chan, Department of Medicine and Therapeutics, 9/F Prince of Wales Hospital, 30-32 Ngan Shing St, Shatin, Hong Kong, China; hlychan@cuhk.edu.hk

Revised version received 17 March 2004 Accepted for publication 21 March 2004
Background: Identification of risk factors for the development of hepatocellular carcinoma (HCC) is important for HCC surveillance in chronic hepatitis B virus (HBV) infection. Our aim was to study the independent risk factors and effect of HBV genotypes on HCC development in a prospective longitudinal cohort of chronic hepatitis B patients.

Patients and methods: Chronic hepatitis B patients recruited since 1997 were prospectively followed up for the development of HCC. HCC was diagnosed by a combination of $\alpha$ fetoprotein, imaging, and histology. Liver cirrhosis was defined as ultrasonic features of cirrhosis together with hypersplenism, ascites, varices, and/or encephalopathy.

Results: In total, 426 patients were followed up for 1664 person years; median 225 (range 12-295) weeks. Forty nine (11\%) patients had underlying clinical liver cirrhosis. A total of $242(57 \%)$ and 179 (42\%) patients had HBV genotypes C and B, respectively. Twenty five patients developed HCC in a median follow up of 121 (range 14-236) weeks. The overall incidence of HCC was 1502 cases per 100000 person years. On multivariate analysis, clinical liver cirrhosis and HBV genotype $\mathrm{C}$ infection were independently associated with HCC development, with an adjusted relative risk of 10.24 (95\% confidence interval (Cl) 4.39-23.89; $p<0.001$ ) and 2.84 (95\% Cl 1.05-7.72; $p=0.040)$, respectively. Patient age, sex, hepatitis B e antigen ( $\mathrm{HBeAg}$ ) status, alanine aminotransferase (ALT) levels, and basal core promoter mutations did not predict HCC development. Patients infected with HBV genotype $\mathrm{C}$ tended to have persistently positive $\mathrm{HBeAg}$ or fluctuating $\mathrm{HBeAg}$ status and higher ALT levels during the follow up period. Conclusion: Genotype C HBV infection is an independent risk factor for HCC development in addition to liver cirrhosis.
E pidemiological studies have shown a strong association between chronic hepatitis B virus (HBV) infection and hepatocellular carcinoma (HCC), which is a major cause of morbidity and mortality in areas where chronic hepatitis B is endemic. ${ }^{1}$ The annual incidence of HCC development in chronic HBV infected patients ranges from $0.2 \%$ to $0.8 \%$ in different centres. ${ }^{2-4}$ Surveillance of HCC by semi annual $\alpha$ fetoprotein testing followed by ultrasound and/or computerised tomography imaging can detect HCC at an early resectable stage, which may be translated to prolongation of patient survival. ${ }^{56}$ As there are 350 million chronic HBV infected patients worldwide, ${ }^{7}$ population based HCC surveillance programmes would imply a huge demand on public health resources. This problem will be more serious in Southeast Asia where the prevalence of chronic hepatitis B is up to $15 \% .{ }^{8}$ Identification of risk factors for HCC and stratification of patient risk is therefore important to guide future surveillance strategy.

Various risk factors for HCC development have been identified but their relationship is complicated. Older patient age and liver cirrhosis have been consistently found to be important risk factors for HCC in most studies. ${ }^{2}{ }^{10}$ However, the incidence of liver cirrhosis also increases with patient age. A population based study in Taiwan found that positive hepatitis B e antigen (HBeAg) at recruitment was associated with a higher risk of HCC development after adjustment for the effect of age and sex after 10 years of follow up. ${ }^{11}$ However, the status of liver cirrhosis was not assessed in this study.

To date, eight different HBV genotypes $(\mathrm{A}-\mathrm{H})$ have been reported according to viral genomic heterogeneity. ${ }^{12-14}$ In Southeast Asia, HBV genotypes $\mathrm{B}$ and $\mathrm{C}$ are most prevalent. ${ }^{15}{ }^{16}$ Case control studies found that HBV genotype $\mathrm{C}$ was associated with a higher risk of HCC than genotype B in Japan and Taiwan. ${ }^{16}{ }^{17}$ Contradictory results were obtained from two large scaled cross sectional studies which found that the distributions of HBV genotypes B and C were similar among patients with and without HCC. ${ }^{18}{ }^{19}$ All of these studies are potentially subject to sampling bias and the potential confounding effects of patient age, HBeAg status, and liver cirrhosis were not assessed. As HBV genotype $\mathrm{C}$ is associated with more aggressive disease than genotype $\mathrm{B}$, HBeAg status and liver cirrhosis status must be taken into consideration before the true impact of HBV genotype on HCC development can be determined. ${ }^{20-22}$ Previous studies have suggested that basal core promoter (BCP) mutations (A to $\mathrm{T}$ at nucleotide 1762 and $\mathrm{G}$ to $\mathrm{A}$ at nucleotide 1764 ), which are commonly found in HBV genotype $\mathrm{C}$, increase the risk of HCC development. ${ }^{23}{ }^{24}$ However, the prevalence of BCP mutations increases with HBeAg seroconversion ${ }^{25}{ }^{26}$ and their association with HCC has not been confirmed in longitudinal studies.

The primary aim of this study was to determine the independent risk factor(s) for HCC development in a prospective longitudinal cohort of chronic hepatitis B patients in Hong Kong. Potential risk factors, including patient demographics, liver cirrhosis, HBeAg status, BCP mutations, and viral genotypes were assessed. The secondary aim was to investigate disease progression and effect on HCC development among patients infected with different HBV genotypes.

Abbreviations: $\mathrm{ALT}$, alanine aminotransferase; anti-HBe, antibody to hepatitis $B$ e antigen; $B C P$, basal core promoter; $\mathrm{HBeAg}$, hepatitis $B$ e antigen; $\mathrm{HBV}$, hepatitis $B$ virus; $H C C$, hepatocellular carcinoma; $P C R$, polymerase chain reaction 


\section{PATIENTS AND METHODS}

\section{Patients}

Consecutive chronic hepatitis B patients of Chinese ethnicity recruited at the Hepatitis Clinic, Prince of Wales Hospital, from December 1997 to July 2000, were studied..$^{25}$ No patient had a history of alcoholism ( $>20 \mathrm{~g} /$ week) or hepatitis $C$ virus coinfection. Patients who had other possible causes of hepatitis or liver cirrhosis, including autoimmune liver disease, primary biliary cirrhosis, Wilson's disease, and haemochromatosis, were also excluded. At the initial presentation, an ultrasound examination of the abdomen was performed to exclude any pre-existing HCC. Patients were prospectively followed up every six months or more frequently if clinically indicated, with monitoring of liver biochemistry, $\mathrm{HBeAg}$, and antibody to hepatitis B e antigen (anti-HBe) status, as well as $\alpha$ fetoprotein levels. Ultrasound of the abdomen, computerised tomography, hepatic angiogram, and/or liver biopsy were performed if $\alpha$ fetoprotein levels were higher than $50 \mu \mathrm{g} / \mathrm{l}$ or demonstrated a rising trend over $20 \mu \mathrm{g} / \mathrm{l}$ to confirm the diagnosis of HCC. For patients with normal $\alpha$ fetoprotein levels, ultrasound of the abdomen was performed every $1-2$ years. Clinical liver cirrhosis was defined as ultrasonic features of liver cirrhosis plus evidence of hypersplenism (splenomegaly with a platelet count of less than $100 \times 10^{9} / 1$ or white count less than $\left.4 \times 10^{9} / 1\right)$, clinical ascites, varices, and/or hepatic encephalopathy.

\section{Serology assays}

HBsAg and anti-hepatitis $\mathrm{C}$ virus antibodies (third generation assay) were tested using commercially available enzyme linked immunosorbant assay kits (Abbott GmBH Diagnostika, Wiesbaden-Delkenheim, Germany). HBeAg and anti-HBe were measured by enzyme linked immunosorbant assay (Sanofi Diagnostics, Pasteur, France).

\section{HBV genotyping}

HBV genotyping was performed in the residual serum sample of each patient at the initial visit. Genotyping polymerase chain reaction (PCR) amplification and restriction fragment length polymorphism were performed as described previously. $^{22} 27$ In short, $10 \mu \mathrm{l}$ of extracted HBV DNA was amplified by PCR using primers flanking the HBV genome between nucleotides 256 to 796 (sense primer 5'-GTG GTG GAC TTC TCT CAA TTT TC and antisense primer 5'-CGG $\mathrm{TA}(\mathrm{A} / \mathrm{T})$ AAA GGG ACT $\mathrm{CA}(\mathrm{A} / \mathrm{C}) \mathrm{GAT})$. The PCR product $(10 \mu \mathrm{l})$ was then mixed and incubated with restriction enzymes Tsp 5091 (New England Biolabs, Beverly, Massachusetts, USA) and HinfI (Boehringer Mannheim, Mannheim, Germany), respectively. After incubation, samples were run on a composite gel containing $2 \%$ NuSieve agarose (FMC BioProducts, Rockland, Maine, USA) and 1\% standard agarose. DNA was visualised by ethidium bromide staining and the restriction pattern was read accordingly.

\section{Basal core promoter mutations}

BCP mutations were determined in the residual serum sample of each patient at the initial visit. PCR and direct sequencing of amplified products was performed as described previously using primers that flanked the core promoter and precore regions. ${ }^{26}$

\section{Statistical analysis}

The Cox proportional hazard model was used to estimate the relative risk of HCC development associated with age, sex, clinical liver cirrhosis, HBeAg status, alanine aminotransferase (ALT) level, BCP mutations, and HBV genotype. Categorical variables were compared by $\chi^{2}$ test and continuous variables by the Mann-Whitney U test. Variables with a $\mathrm{p}$ value $<0.1$ on univariate analysis were analysed by backward stepwise multivariate analysis for independent risk factors associated with HCC development. The 95\% confidence interval (95\% CI) for the relative risks was also calculated. Cumulative probability of HCC development was determined by the Kaplan-Meier method and compared by log rank test. All statistical analyses were performed using the Statistical Package of Social Science (SPSS version 11.0). All statistical tests were two sided. Statistical significance was taken as $\mathrm{p}<0.05$.

\section{RESULTS}

\section{Baseline characteristics}

A total of 426 patients, including 278 males and 148 females (median age 41 (range 12-80) years) were followed up for 1664 person years (median 225 (range 12-295) weeks). Forty nine $(11 \%)$ patients had underlying clinical liver cirrhosis. Twenty nine (59\%) patients had Child's A, 15 (31\%) Child's $\mathrm{B}$, and five (10\%) patients had Child's C cirrhosis. Baseline clinical data are shown in table 1 . Two hundred and forty two (57\%) patients had HBV genotype C, 179 (42\%) had genotype $\mathrm{B}$, three had a mixed genotype $\mathrm{B}$ and $\mathrm{C}$, one patient had genotype A, and one patient had negative PCR. A total of 215 $(56 \%)$ patients had BCP mutations; among them two patients had deletion of nucleotides 1762 and 1764 .

\section{Risk factors of HCC}

A total of 25 patients developed HCC in a median follow up of time of 121 (range 14-236) weeks. The overall incidence of HCC was 1502 cases per 100000 person years. The cumulative probability of HCC development was $1.4 \%$, $2.9 \%, 4.5 \%, 5.9 \%$, and $6.9 \%$ from the first to the fifth year

Table 1 Univariate analysis for factors associated with development of hepatocellular carcinoma (HCC)

\begin{tabular}{lclcr}
\hline & Overall & HCC & No HCC & p Value \\
\hline $\mathrm{n}$ & 426 & 25 & 401 & - \\
Age (y) (mean (SD)) & $41(13)$ & $52(10)$ & $40(13)$ & $<0.001$ \\
Age $>40(\mathrm{n}(\%))$ & $228(54 \%)$ & $21(84 \%)$ & $207(52 \%)$ & 0.006 \\
Male (n (\%)) & $278(65 \%)$ & $21(84 \%)$ & $257(64 \%)$ & 0.057 \\
Cirrhosis (n (\%)) & $49(11 \%)$ & $14(56 \%)$ & $35(9 \%)$ & $<0.001$ \\
HBeAg+ (n (\%)) & $157(37 \%)$ & $10(40 \%)$ & $147(37 \%)$ & 0.76 \\
ALT & $50(12-1072)$ & $64(41-283)$ & $49(12-1072)$ & 0.45 \\
Genotype C (n (\%))* & $242(57 \%)$ & $18(75 \%)$ & $224(56 \%)$ & 0.089 \\
BCP mutations (n (\%))† & $215(56 \%)$ & $19(83 \%)$ & $196(54 \%)$ & 0.017 \\
Follow up (weeks) & $225(12-295)$ & $121(14-236)$ & $226(12-295)$ & - \\
\hline
\end{tabular}

*Five patients were not evaluated; one patient who developed HCC had a mixed HBV genotype B and C. tForty three patients (two had HCC) had a negative polymerase chain reaction (PCR) for BCP mutations and were not evaluated. Twenty patients had genotype B, 21 patients had genotype $C$, one patient had a mixed genotype $B$ and $C$, and one patient had a negative PCR for HBV genotyping

$\mathrm{HBeAg}$, hepatitis B e antigen; ALT, alanine aminotransferase; BCP, basal core promoter. 


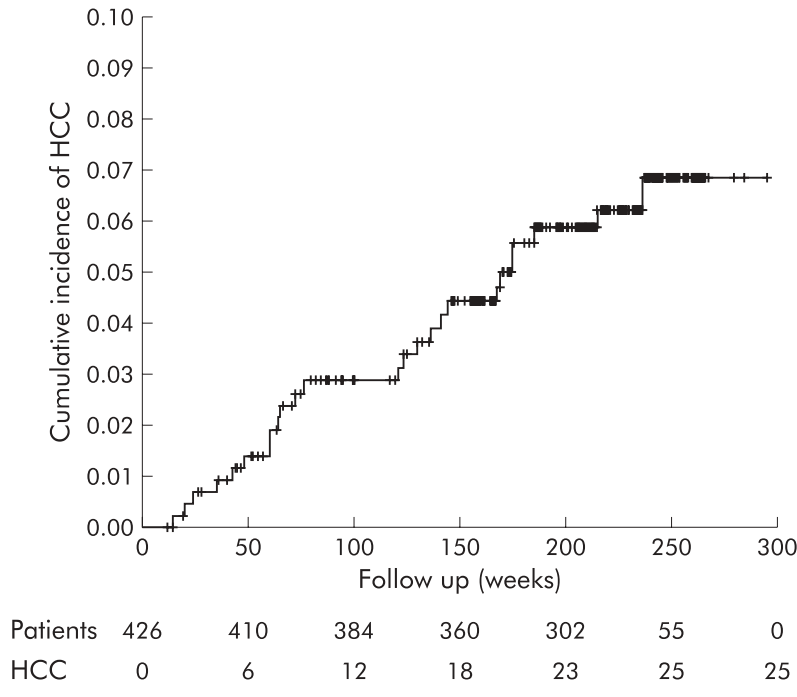

Figure 1 Cumulative incidence of hepatocellular carcinoma (HCC) in the studied cohort.

of follow up (fig 1). On univariate analysis of baseline variables, age $>40$ years, male sex, presence of clinical liver cirrhosis, BCP mutations, and HBV genotype C were associated with the development of HCC $(p<0.1)$ (table 1$)$. HBeAg positivity and ALT levels were not associated with HCC development.

On multivariate analysis, clinical liver cirrhosis and genotype $\mathrm{C} \mathrm{HBV}$ infection were independently associated with HCC development. Clinical liver cirrhosis was the strongest predictor of HCC development with an adjusted relative risk of 10.24 (95\% CI 4.39-23.89; p<0.001) whereas HBV genotype C has an adjusted relative risk of 2.84 (95\% CI $1.05-7.72 ; \mathrm{p}=0.040$ ). On subgroup analysis, the incidence of HCC development among patients with clinical cirrhosis was 1.1-fold higher in HBV genotype C ( 10608 cases per 100000 person years) versus HBV genotype B (5147 cases per 100000 person years) infection $(p=0.21)$ (fig 2). Similarly, the incidence of HCC development among patients who had no clinical liver cirrhosis was 2.3-fold higher in HBV genotype C ( 1049 cases per 100000 person years) versus HBV genotype B (322 cases per 100000 person years) infection $(\mathrm{p}=0.11)$ (fig 2). Differences in incidence of HCC development were however not statistically significant between HBV genotypes $\mathrm{B}$ and $\mathrm{C}$ with or without liver cirrhosis, probably due to the small number of events in each subgroup and the marginal significance of HBV genotype.

Mean (SD) age of HCC development was comparable between patients infected with HBV genotype B (49 (15)) and genotype $C(53(9))(p=0.51)$. Using age 40 years as the cut off, there was no difference in the proportion of patients infected with HBV genotype C for early (2/4 patients, 50\%) and late $(16 / 18$ patients, $89 \%)$ onset HCC $(p=0.21)$.

\section{HCC in non-cirrhotic livers}

Among 11 patients who developed HCC but without clinical evidence of liver cirrhosis, nine had liver histology available. Seven $(78 \%)$ patients had histological evidence of severe fibrosis or liver cirrhosis while the remaining two patients had mild to moderate liver fibrosis (not to the stage of liver cirrhosis). Hence at least $8 \%(2 / 25)$ of the HCC developed in our cohort were derived from a non-cirrhotic liver. These two patients were male, aged 36 and 49 years, respectively, infected with HBV genotype C. They had mildly elevated ALT (2-5 times the upper limit of the laboratory normal) and

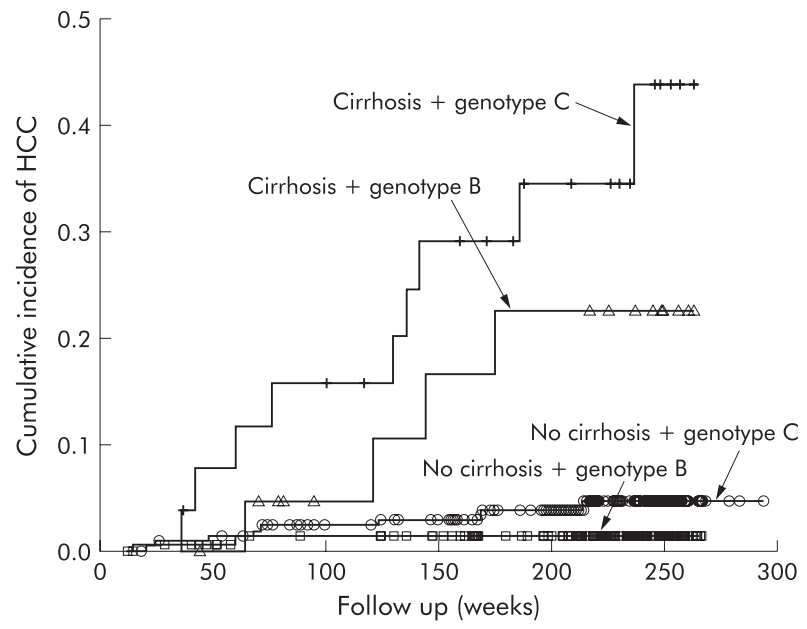

Figure 2 Cumulative incidence of hepatocellular carcinoma $(\mathrm{HCC})$ with reference to liver cirrhosis status and hepatitis $B$ virus (HBV) genotype. Log rank test comparing the cumulative incidence of $\mathrm{HCC}$ among the four studied groups, $p<0.001$.

persistently negative HBeAg during a follow up period of 123 and 169 weeks, respectively, before HCC was diagnosed. Serum HBV DNA levels at initial visits were 1770000 and 800 copies/ml, respectively.

\section{Disease patterns of different HBV genotypes}

At baseline, patients infected with $\mathrm{HBV}$ genotype $\mathrm{C}$ were generally younger with a borderline female predominance and higher proportion of positive HBeAg compared with those infected with HBV genotype B (table 2). The prevalence of BCP mutations was higher among patients infected with genotype C. Otherwise, the percentage of clinical liver cirrhosis, median ALT levels, and duration of follow up were comparable between patients infected with HBV genotypes B and $\mathrm{C}$.

On follow up, 87 of 242 (36\%) patients infected with HBV genotype $C$ versus 33 of $179(18 \%)$ infected with HBV genotype B had persistently positive HBeAg or fluctuating HBeAg status $(\mathrm{p}<0.001)$ (table 2 ). In contrast 123 of 242 (51\%) patients infected with HBV genotype C versus 127 of $179(71 \%)$ infected with genotype B had persistently negative HBeAg $(p<0.001)$ (table 2). A similar proportion of patients infected with both HBV genotypes underwent sustained HBeAg seroconversion. In general, HBV genotype C was associated with higher ALT levels than genotype B $(\mathrm{p}=0.015)$ (table 2).

\section{DISCUSSION}

The results of this study confirm that HBV genotype $\mathrm{C}$ is associated with an increased risk of HCC, and is independent of the presence of liver cirrhosis. Patient age, sex, HBeAg positivity, and ALT levels had no independent impact on the cumulative probability of HCC development after five years of follow up.

The overall incidence of HCC in this study was 1052 cases per 100000 person years. The high incidence of HCC in this study may be related to the $11 \%$ of patients who had clinical liver cirrhosis with a very high incidence of HCC (7998 cases per 100000 person years). If only non-cirrhotic patients are considered, and incidence of HCC was 743 cases per 100000 person years. This is comparable with the reported incidence in Taiwan (822 per 100000 person years) but still higher than that reported in Toronto, Canada (470 per 100000 person years). ${ }^{3}$ One reason may be related to the long 
Table 2 Clinical characteristics of patients infected with hepatitis B virus (HBV) genotypes $B$ and $C$

\begin{tabular}{lccc}
\hline & Genotype B & Genotype C & p Value \\
\hline Baseline characteristics & 179 & & \\
n & $43(14)$ & 242 & \\
Age (y) (mean (SD)) & $106(60 \%)$ & $116(13)$ & 0.005 \\
Age > 40 y (n (\%)) & $124(71 \%)$ & $150(62 \%)$ & 0.012 \\
Male (n (\%)) & $22(12 \%)$ & $26(11 \%)$ & 0.095 \\
Cirrhosis (n (\%)) & $47(27 \%)$ & $110(46 \%)$ & $<0.02$ \\
HBeAg+ (n (\%)) & $46(12-1072)$ & $54(13-1011)$ & 0.075 \\
ALT & $62(39 \%)$ & $151(68 \%)$ & $<0.001$ \\
BCP mutationst (n (\%)) & $226(12-266)$ & $224(19-295)$ & 0.58 \\
Follow up (weeks) & $20(11 \%)$ & $54(22 \%)$ & 0.005 \\
HBeAg trend on follow up & $13(7 \%)$ & $33(14 \%)$ & 0.055 \\
Persistently positive HBeAg (n (\%)) & $19(11 \%)$ & $32(13 \%)$ & 0.51 \\
Fluctuating HBeAg status (n (\%)) & $127(71 \%)$ & $123(51 \%)$ & $<0.001$ \\
Sustained HBeAg seroconversion (n (\%)) & $105(59 \%)$ & $115(47 \%)$ & 0.015 \\
Persistently negative HBeAg (n (\%)) & $59(33 \%)$ & $94(39 \%)$ & \\
Peak ALT on follow up (n (\%)) & $15(8 \%)$ & $33(14 \%)$ & \\
$\quad$ <2×ULN & &
\end{tabular}

$B C P$, basal core promoter; $\mathrm{HBeAg}$, hepatitis $B$ e antigen; $A L T$, alanine aminotransferase; ULN, upper limit of normal.

†Twenty patients with genotype $B$ and 21 patients with genotype $C H B V$ infection had negative a polymerase chain reaction for $\mathrm{BCP}$ mutations and were not evaluated.

history of chronic hepatitis B among Asian patients who usually acquire the infection at early infancy. Different viral genotypes between Asian and Canadian patients may be another contributing factor for the observed difference. As in all other studies, the possibility of occult HCC, particularly among patients who had cirrhotic liver, could not be excluded.

In this prospective study, clinical liver cirrhosis was the most important independent risk factor for HCC development. As liver cirrhosis is defined by clinical criteria, all patients in this category have established liver cirrhosis while patients with early liver cirrhosis may be classified as noncirrhotic. This explains the relatively high cumulative incidence of HCC among cirrhotic patients in this study compared with previous series among compensated cirrhotic patients. ${ }^{10}$ The use of clinical criteria to classify liver cirrhosis enables an undisputable diagnosis of liver cirrhosis while the diagnosis of early liver cirrhosis on histology may be affected by biopsy size and interobserver variations. ${ }^{28}$ As liver biopsy may not be a routine procedure for all chronic hepatitis B patients, particularly among those who have normal liver enzymes as in our centre, a clinical diagnosis is more feasible in clinical practice.

Patients who have developed liver cirrhosis are usually older, and male patients generally have more advanced liver disease than female patients. ${ }^{9}{ }^{29}$ The same association also holds true in the case of HCC, as shown in the univariate analysis. When liver cirrhosis was included in the multivariate analysis equation, the impact of these two demographic factors on HCC development became insignificant. Therefore, in surveillance programmes for HCC, the importance of patient age and sex should not be overemphasised as long as the status of clinical liver cirrhosis is taken into consideration.

HBV genotype $C$ was found to be an independent risk factor for HCC development, which concurs with results of case control studies reported previously. ${ }^{15}{ }^{16}$ This association was not as obvious on univariate analysis, probably due to the younger age and marginal female predominance among patients infected with HBV genotype C. Although BCP mutations are strongly associated with HBV genotype $\mathrm{C}^{21}{ }^{23}$ no association between BCP mutations and HCC development was found in this study on multivariate analysis. More patients infected with HBV genotype $\mathrm{C}$ had persistently positive $\mathrm{HBeAg}$ and fluctuating $\mathrm{HBeAg}$ whereas more patients infected with genotype B had persistently negative HBeAg on follow up. This may reflect a relatively higher level of viraemia among patients infected with HBV genotype C. ${ }^{30}{ }^{31}$ One of two patients who developed HCC from a noncirrhotic liver in this study had elevated HBV DNA levels despite persistently negative HBeAg on follow up. Viral genotype and high viraemia may be an independent direct cause of hepatocarcinogenesis in addition to liver cirrhosis, which is the result of continuous necroinflammation. ${ }^{32}$ The direct carcinogenic mechanism may act through increasing cis activation of the proto-oncogene, suppression of tumour suppressor gene, or transactivation by HBV X protein. ${ }^{33}{ }^{34}$

In contrast with the Taiwan report, a single HBeAg result at baseline could not predict the risk of HCC development in this study. ${ }^{11}$ One probable reason is that a significant proportion of HBeAg positive patients underwent HBeAg seroconversion while some $\mathrm{HBeAg}$ negative patients had HBeAg reversion during follow up. In fact, none of the 51 patients who underwent sustained HBeAg seroconversion developed HCC in the present cohort. This concurs with previous reports that sustained HBeAg seroconversion is associated with a favourable prognosis. ${ }^{25}$ In contrast, fluctuating HBeAg status may associate with more aggressive disease progression although it was not confirmed in this study (4/25 (11\%) with HCC versus $42 / 401$ (16\%) without HCC; $p=0.60){ }^{2}$ In this study, the impact of baseline HBV DNA level on HCC development was not evaluated. As HBV DNA is closely related to HBeAg status and tends to change with time, particularly on $\mathrm{HBeAg}$ seroconversion, serial monitoring of HBV DNA is necessary. ${ }^{30} 31$

In summary, $\mathrm{HBV}$ genotype $\mathrm{C}$ is an independent risk factor for the development of HCC in addition to liver cirrhosis. Viral genotype should therefore be determined on risk stratification in future HCC surveillance programmes. The exact reason for the higher risk of hepatocarcinogenesis associated with HBV genotype C warrants further investigation but direct viral effects in addition to necroinflammation and liver cirrhosis are possible.

\section{ACKNOWLEDGEMENTS}

We would like to thank the Cheng Suen Man Shook Foundation for the Study of Viral Hepatitis for support of this study. 


\section{Authors' affiliations}

H L-Y Chan, A Y Hui, M L Wong, A M-L Tse, L C-T Hung, V W-S Wong, J J-Y Sung, Department of Medicine and Therapeutics, Chinese University of Hong Kong, Hong Kong SAR, China

\section{REFERENCES}

1 Beasley RP, Lin CC, Hwang LY, et al. Hepatocellular carcinoma and hepatitis B virus. A prospective study of 22707 men in Taiwan. Lancet 1981 ;ii: 1129-32.

2 McMahon BJ, Holck P, Bulkow L, et al. Serologic and clinical outcomes of 1536 Alaska natives chronically infected with hepatitis B virus. Ann Intern Med 2001;135:759-68.

3 Liaw YF, Tai DI, Chu CM, et al. Early detection of hepatocellular carcinoma in patients with chronic type B hepatitis. A prospective study. Gastroenterology 1986;90:263-7.

4 Sherman M, Peltekian KM, Lee C. Screening for hepatocellular carcinoma in chronic carriers of hepatitis $B$ virus: incidence and prevalence of hepatocellular carcinoma in a North American urban population. Hepatology 1995;22:432-8

5 McMahon BJ, Bulkow L, Harpster A, et al. Screening for hepatocellular carcinoma in Alsaka natives infected with chronic hepatitis B: A 16-year population-based study. Hepatology 2000;32:842-6.

6 Yuen MF, Cheng CC, Lauder IJ, et al. Early detection of hepatocellular carcinoma increases the chance of treatment: Hong Kong experience. Hepatology 2000;31:330-5.

7 Lee WM. Hepatitis B virus infection. N Engl J Med 1997:337:1733-45.

8 Gust ID. Epidemiology of hepatitis B infection in the Western Pacific and South East Asia. Gut 1996;38(suppl 2):S18-23.

9 Liaw YF, Chu CM, Lin DY, et al. Age-specific prevalence and significance of hepatitis $B$ e antigen and antibody in chronic hepatitis $B$ virus infection in Taiwan: A comparison among asymptomatic carriers, chronic hepatitis, liver cirrhosis, and hepatocellular carcinoma. J Med Virol 1984;13:385-91.

10 Fattovich G, Giustina G, Schalm SW, et al. Occurrence of hepatocellular carcinoma and decompensation in Western European patients with cirrhosis type B. Hepatology 1995;21:77-82.

11 Yang HI, Lu SN, Liaw YF, et al. Hepatitis B e antigen and the risk of hepatocellular carcinoma. N Engl J Med 2002;347:168-74.

12 Norder H, Courouce AM, Magnius LO. Complete genomes, phylogenetic relatedness, and structural proteins of six strains of the hepatitis B virus, four of which represent two new genotypes. Virology 1994;198:489-503.

13 Stuyver L, De Gendt S, Van Geyt C, et al. A new genotype of hepatitis B virus: complete genome and phylogenetic relatedness. J Gen Virol 2000;81:67-74.

14 Arauz-Ruiz P, Norder H, Robertson BH, et al. Genotype H: a new Amerindian genotype of hepatitis B virus revealed in Central America. J Gen Virol 2002;83:2059-73.

15 Orito E, Ichida T, Sakugawa H, et al. Geographic distribution of hepatitis B virus (HBV) genotype in patients with chronic HBV infection in Japan. Hepatology 2001;34:590-4.

$16 \mathrm{Kao} \mathrm{JH}$, Chen PJ, Lai MY, et al. Hepatitis B genotypes correlate with clinical outcome in patients with chronic hepatitis B. Gastroenterology 2000;1 18:554-9.
17 Fujie $\mathrm{H}$, Moriya $\mathrm{K}$, Shintani $\mathrm{Y}$, et al. Hepatitis B virus genotype and hepatocellular carcinoma in Japan. Gastroenterology 2001;120:1564-5.

18 Sumi H, Yokosuka O, Seki N, et al. Influence of hepatitis B virus genotype on the progression of chronic type B liver disease. Hepatology 2003;37:19-26.

19 Yuen MF, Sablon E, Yuan HJ, et al. Significance of hepatitis B genotype in acute exacerbation, $\mathrm{HBeAg}$ seroconversion, cirrhosis-related complications, and hepatocellular carcinoma. Hepatology 2003;37:562-7.

20 Chu CJ, Hussain M, Lok ASF. Hepatitis B virus genotype B is associated with earlier $\mathrm{HBeAg}$ seroconversion compared with hepatitis $B$ virus genotype $\mathrm{C}$. Gastroenterology 2002;122:1756-62.

21 Chan HLY, Tsang SWC, Liew CT, et al. Viral genotype and hepatitis B virus DNA levels are correlated with histological liver damage in $\mathrm{HBeAg}$-negative chronic hepatitis B virus infection. Am J Gastroenterol 2002;97:406-12.

22 Chan HLY, Wong ML, Hui AY, et al. Genotype $C$ hepatitis B virus takes a more aggressive disease course before hepatitis $B$ e antigen seroconversion as compared to genotype B hepatitis B virus. J Clin Microbiol 2003;41:1277-9.

$23 \mathrm{Kao} \mathrm{JH}$, Chen PJ, Lai MY, et al. Basal core promoter mutations of hepatitis $B$ virus increase the risk of hepatocellular carcinoma in hepatitis $B$ carriers. Gastroenterology 2003;124:327-34.

24 Baptista M, Kramvis A, Kew MC. High prevalence of 1762(T) 1764(A) mutations in the basic core promoter of hepatitis $B$ virus isolated from black Africans with hepatocellular carcinoma compared with asymptomatic carriers. Hepatology 1999;29:946-53.

25 Chan HLY, Leung NWY, Hussain M, et al. Hepatitis B e antigen-negative chronic hepatitis B in Hong Kong. Hepatology 2000;31:763-8.

26 Chan HLY, Hussain M, Lok ASF. Different hepatitis B virus genotypes are associated with different mutations in the core promoter and precore regions during hepatitis B e antigen seroconversion. Hepatology 1999;29:976-84.

27 Lindh M, Andersson AS, Gusdal A, et al. 1858 variants, and geographical origin of hepatitis $B$ virus-large-scaled analysis using a new genotyping method. J Infect Dis 1997; 175:1285-93.

28 Colloredo G, Guido M. Sonzongni A et al. Impact of liver biopsy size on histological evaluation of chronic viral hepatitis: the smaller the sample, the milder the disease. J Hepatol 2003;39:239-44.

29 Lok AS. Natural history and control of perinatally acquired hepatitis B virus infection. Dig Dis 1992;10:46-52.

30 Chan HLY, Wong ML, Hui AY, et al. The value of hepatitis $B$ virus DNA quantitation to predict hepatitis $B$ e antigen reversion in chronic hepatitis $B$. J Clin Microbiol 2003;41:4693-5.

31 Chu CJ, Hussain M, Lok ASF. Quantitative serum HBV DNA levels during different stages of chronic hepatitis B infection. Hepatology 2003:36:1408-15.

32 Ohkubo K, Kato Y, Ichikawa T, et al. Viral load is a significant prognostic factor for hepatitis B virus-associated hepatocellular carcinoma. Cancer 2002;94:2663-8

33 Shafritz DA, Kew MC. Identification of integrated hepatitis B virus DNA sequences in human hepatocellular carcinoma. Hepatology 1981;1:1-8.

34 Matsubara K, Tokino T. Integration of hepatitis B virus DNA and its implications for hepatocarcanogenesis. Mol Biol Med 1990;7:243-60.

35 Hsu YS, Chien RN, Yeh CT, et al. Long-term outcome after spontaneous $\mathrm{HBeAg}$ seroconversion in patients with chronic hepatitis B. Hepatology 2002;35:1522-7.

\section{EDITOR'S QUIZ: GI SNAPSHOT}

\section{Answer}

From question on page 1484

The thoracoabdominal computed tomography scan revealed the presence of liquid and bowel in the left hemithorax. Parietal thickening and enhancement strongly suggested bowel infarction. The heart and mediastinum were shifted to the right side. An exploratory laparotomy was performed. The oesophageal hiatus was normal but a $4.5 \mathrm{~cm}$ rent was discovered in the posterior leaf of the left hemi diaphragm through which herniated a massively dilated transverse colon and jejunum, with evidence of infarction. Transverse colectomy and resection of $2 \mathrm{~m}$ of jejunum were performed with direct diaphragm repair. A chest tube was placed in the left hemithorax. The postoperative course was uneventful. The patient was discharged from hospital 10 days after surgery.

One should be aware of the possibility of diaphragmatic hernia in patients with a known history of abdominal trauma. Although uncommon, strangulation of the colon and small bowel through a rent in the diaphragm should be considered when there is radiological evidence of herniation. 